\title{
Low Probability of Intercept Triangular Modulated Frequency Modulated Continuous Wave Signal Characterization Comparison using the Spectrogram and the Scalogram
}

Abstract- Digital intercept receivers are currently moving away from Fourier-based analysis and towards classical time-frequency analysis techniques for the purpose of analyzing low probability of intercept radar signals. This paper presents the novel approach of characterizing low probability of intercept frequency modulated continuous wave radar signals through utilization and direct comparison of the Spectrogram versus the Scalogram. Two different triangular modulated frequency modulated continuous wave signals were analyzed. The following metrics were used for evaluation: percent error of: carrier frequency, modulation bandwidth, modulation period, chirp rate, and time-frequency localization ( $x$ and $y$ direction). Also used were: percent detection, lowest signal-to-noise ratio for signal detection, and plot (processing) time. Experimental results demonstrate that overall, the Spectrogram produced more accurate characterization metrics than the Scalogram. An improvement in performance may well translate into saved equipment and lives.

GJRE-F Classification: FOR Code: 090609

Strictly as per the compliance and regulations of :

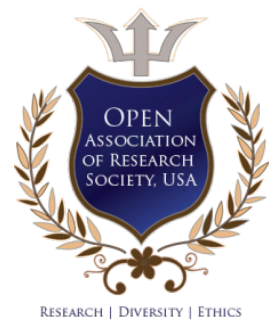

(c) 2017. Daniel L. Stevens \& Stephanie A. Schuckers. This is a research/review paper, distributed under the terms of the Creative Commons Attribution-Noncommercial 3.0 Unported License http://creativecom mons.org/ licenses/by-nc/3.0/), permitting all non commercial use, distribution, and reproduction in any medium, provided the original work is properly cited. 


\title{
Low Probability of Intercept Triangular Modulated Frequency Modulated Continuous Wave Signal Characterization Comparison using the Spectrogram and the Scalogram ${ }^{1}$
}

\author{
Daniel L. Stevens ${ }^{\alpha}$ \& Stephanie A. Schuckers ${ }^{\sigma}$
}

Abstract-Digital intercept receivers are currently moving away from Fourier-based analysis and towards classical timefrequency analysis techniques for the purpose of analyzing low probability of intercept radar signals. This paper presents the novel approach of characterizing low probability of intercept frequency modulated continuous wave radar signals through utilization and direct comparison of the Spectrogram versus the Scalogram. Two different triangular modulated frequency modulated continuous wave signals were analyzed. The following metrics were used for evaluation: percent error of: carrier frequency, modulation bandwidth, modulation period, chirp rate, and time-frequency localization ( $x$ and $y$ direction). Also used were: percent detection, lowest signal-to-noise ratio for signal detection, and plot (processing) time. Experimental results demonstrate that overall, the Spectrogram produced more accurate characterization metrics than the Scalogram. An improvement in performance may well translate into saved equipment and lives.

\section{InTRODUCTION}

rad requency Modulated Continuous Wave (FMCW) signals are frequently encountered in modern radar systems [WAN10], [WON09], [WAJ08]. The frequency modulation spreads the transmitted energy over a large modulation bandwidth $\Delta \mathrm{F}$, providing good range resolution that is critical for discriminating targets from clutter. The power spectrum of the FMCW signal is nearly rectangular over the modulation bandwidth, so non-cooperative interception is difficult. Since the transmit waveform is deterministic, the form of the return signals can be predicted. This gives it the added advantage of being resistant to interference (such as jamming), since any signal not matching this form can be suppressed [WIL06]. Consequently, it is difficult for an intercept receiver to detect the FMCW waveform and measure the parameters accurately enough to match the jammer waveform to the radar waveform [PAC09].

The most popular linear modulation utilized is the triangular FMCW emitter [LIA09], since it can

Author a: Air Force Research Laboratory Rome, NY 13441. e-mail: daniel.stevens.7@us.af.mil

Auhtor 6: Department of Electrical and Computer Engineering, Clarkson University Potsdam, NY 13699. e-mail: sschucke@clarkson.edu

\footnotetext{
${ }^{1}$ Approved for Public Release; Distribution Unlimited: CaseNumber: 88ABW-2017-1873, 25-Apr-2017
}

measure the target's range and Doppler [MIL02], [LIW08]. Triangular modulated FMCW is the waveform that is employed in this paper.

Time-frequency signal analysis involves the analysis and processing of signals with time-varying frequency content. Such signals are best represented by a time-frequency distribution [PAP95], [HAN00], which is intended to show how the energy of the signal is distributed over the two-dimensional time-frequency plane [WEI03], [LIX08], [OZD03]. Processing of the signal may then exploit the features produced by the concentration of signal energy in two dimensions (time and frequency), instead of only one dimension (time or frequency) [BOA03], [LIY03]. Since noise tends to spread out evenly over the time-frequency domain, while signals concentrate their energies within limited time intervals and frequency bands; the local SNR of a noisy signal can be improved simply by using time-frequency analysis [XIA99]. Also, the intercept receiver can increase its processing gain by implementing timefrequency signal analysis [GUL08].

Time-frequency distributions are useful for the visual interpretation of signal dynamics [RAN01]. An experienced operator can quickly detect a signal and extract the signal parameters by analyzing the timefrequency distribution [ANJ09].

The Spectrogram is defined as the magnitude squared of the Short-Time Fourier Transform (STFT) [HIP00], [HLA92], [MIT01], [PAC09], [BOA03]. For nonstationary signals, the STFT is usually in the form of the Spectrogram [GRI08].

The STFT of a signal $x(u)$ is given in equation 1 as:

$$
F_{x}(t, f ; h)=\int_{-\infty}^{+\infty} x(u) h(u-t) e^{-j 2 \pi f u} d u
$$

Where $h(t)$ is a short time analysis window localized around $\mathrm{t}=0$ and $\mathrm{f}=0$. Because multiplication by the relatively short window $\mathrm{h}(\mathrm{u}-\mathrm{t})$ effectively suppresses the signal outside a neighborhood around the analysis point $u=t$, the STFT is a 'local' spectrum of the signal $x(u)$ around $t$. Think of the window $h(t)$ as sliding along the signal $x(u)$ and for each shift $h(u-t)$ we compute the usual Fourier transform of the product function $x(u) h(u-t)$. The observation window allows 
localization of the spectrum in time, but also smears the spectrum in frequency in accordance with the uncertainty principle, leading to a trade-off between time resolution and frequency resolution. In general, if the window is short, the time resolution is good, but the frequency resolution is poor, and if the window is long, the frequency resolution is good, but the time resolution is poor.

The STFT was the first tool devised for analyzing a signal in both time and frequency simultaneously. For analysis of human speech, the main method was, and still is, the STFT. In general, the STFT is still the most widely used method for studying non-stationary signals [COH95].

The Spectrogram (the squared modulus of the STFT) is given by equation 2 as:

$$
S_{x}(t, f)=\left|\int_{-\infty}^{+\infty} x(u) h(u-t) e^{-j 2 \pi f u} d u\right|^{2}
$$

The Spectrog ram is a real-valued and nonnegative distribution. Since the window $h$ of the STFT is assumed of unit energy, the Spectrogram satisfies the global energy distribution property. Thus we can interpret the Spectrogram as a measure of the energy of the signal contained in the time-frequency domain centered on the point ( $t, f)$ and whose shape is independent of this localization.

Here are some properties of the Spectrogram:

1) Time and Frequency covariance- The Spectrogram preserves time and frequency shifts, thus the spectrogram is an element of the class of quadratic time-frequency distributions that are covariant by translation in time and in frequency (i.e. Cohen's class);

2) Time-Frequency Resolution- The time-frequency resolution of the Spectrogram is limited exactly as it is for the STFT; there is a trade-off between time resolution and frequency resolution;

3) Interference Structure- As it is a quadratic (or bilinear) representation, the Spectrogram of the sum of two signals is not the sum of the two Spectrograms (quadratic superposition principle); there is a cross-Spectrogram part and a real part. Thus, as for every quadratic distribution, the Spectrogram presents interference terms; however, those interference terms are restricted to those regions of the time-frequency plane where the signals overlap. Thus if the signal components are sufficiently distant so that their Spectrograms do not overlap significantly, then the interference term will nearly be identically zero [ISI96], [COH95], [HLA92].

The Scalogram is defined as the magnitude squared of the wavelet transform, and can be used as a time-frequency distribution [COH02], [GAL05], [BOA03].

The idea of the wavelet transform (equation (3)) is to project a signal $x$ on a family of zero-mean functions (the wavelets) deduced from an elementary function (the mother wavelet) by translations and dilations:

$$
T_{x}(t, a ; \Psi)=\int_{-\infty}^{+\infty} x(s) \Psi_{t, a}^{*}(s) d s
$$

where $\quad \Psi_{t, a}(s)=|a|^{-1 / 2} \Psi\left(\frac{s-t}{a}\right)$. The variable a corresponds to a scale factor, in the sense that taking $|a|>1$ dilates the wavelet $\Psi$ and taking $|a|<1$ compresses $\Psi$. By definition, the wavelet transform is more a time-scale than a time-frequency representation. However, for wavelets which are well localized around a non-zero frequency $v \_0$ at a scale $=1$, a time-frequency interpretation is possible thanks to the formal identification $v=\frac{v_{0}}{a}$.

The wavelet transform is of interest for the analysis of non-stationary signals, because it provides still another alternative to the STFT and to many of the quadratic time-frequency distributions. The basic difference between the STFT and the wavelet transform is that the STFT uses a fixed signal analysis window, whereas the wavelet transform uses short windows at high frequencies and long windows at low frequencies. This helps to diffuse the effect of the uncertainty principle by providing good time resolution at high frequencies and good frequency resolution at low frequencies. This approach makes sense especially when the signal at hand has high frequency components for short durations and low frequency components for long durations. The signals encountered in practical applications are often of this type.

The wavelet transform allows localization in both the time domain via translations of the mother wavelet, and in the scale (frequency) domain via dilations. The wavelet is irregular in shape and compactly supported, thus making it an ideal tool for analyzing signals of a transient nature; the irregularity of the wavelet basis lends itself to analysis of signals with discontinuities or sharp changes, while the compactly supported nature of wavelets enables temporal localization of a signal's features [BOA03]. Unlike many of the quadratic functions such as the Wigner-Ville Distribution (WVD) and Choi-Williams Distribution (CWD), the wavelet transform is a linear transformation, therefore cross-term interference is not generated. There is another major difference between the STFT and the wavelet transform; the STFT uses sines and cosines as an orthogonal basis set to which the signal of interest is effectively correlated against, whereas the wavelet transform uses special 'wavelets' which usually comprise an orthogonal basis set. The wavelet transform then computes coefficients, which represents a measure of the similarities, or correlation, of the signal with respect to the set of wavelets. In other words, the wavelet transform of a signal corresponds to its decomposition with respect to a family of functions obtained by dilations (or 
contractions) and translations (moving window) of an analyzing wavelet.

A filter bank concept is often used to describe the wavelet transform. The wavelet transform can be interpreted as the result of filtering the signal with a set of bandpass filters, each with a different center frequency [GRI08], [FAR96], [SAR98], [SAT98].

Like the design of conventional digital filters, the design of a wavelet filter can be accomplished by using a number of methods including weighted least squares [ALN00], [GOH00], orthogonal matrix methods [ZAH99], nonlinear optimization, optimization of a single parameter (e.g. the passband edge) [ZHA00], and a method that minimizes an objective function that bounds the out-of-tile energy [FAR99].

Here are some properties of the wavelet transform: 1) The wavelet transform is covariant by translation in time and scaling. The corresponding group of transforms is called the Affine group; 2) The signal $x$ can be recovered from its wavelet transform via the synthesis wavelet; 3) Time and frequency resolutions, like in the STFT case, are related via the Heisenberg-Gabor inequality. However in the wavelet transform case, these two resolutions depend on the frequency: the frequency resolution becomes poorer and the time resolution becomes better as the analysis frequency grows; 4) Because the wavelet transform is a linear transform, it does not contain cross-term interferences [GRI07], [LAR92].

A similar distribution to the Spectrogram can be defined in the wavelet case. Since the wavelet transform behaves like an orthonormal basis decomposition, it can be shown that it preserves energy:

$$
\iint_{-\infty}^{+\infty}\left|T_{x}(t, a ; \Psi)\right|^{2} d t \frac{d a}{a^{2}}=E_{x}
$$

where $E_{x}$ is the energy of $x$. This leads us to define the Scalogram (equation (4)) of $x$ as the squared modulus of the wavelet transform. It is an energy distribution of the signal in the time-scale plane, associated with the measure $\frac{d a}{a^{2}}$.

As is the case for the wavelet transform, the time and frequency resolutions of the Scalogram are related via the Heisenberg-Gabor principle.

The interference terms of the Scalogram, as for the spectrogram, are also restricted to those regions of the time-frequency plane where the corresponding signals overlap. Therefore, if two signal components are sufficiently far apart in the time-frequency plane, their cross-Scalogram will be essentially zero [ISI96], [HLA92].

For this paper, the Morlet Scalogram will be used. The Morlet wavelet is obtained by taking a complex sine wave and by localizing it with a Gaussian envelope. The Mexican hat wavelet isolates a single bump of the Morlet wavelet. The Morlet wavelet has good focusing in both time and frequency [CHE09].

\section{Methodology}

The methodologies detailed in this section describe the processes involved in obtaining and comparing metrics between the classical time-frequency analysis techniques of the Spectrogram and the Scalogram for the detection and characterization of low probability of intercept triangular modulated FMCW radar signals.

The tools used for this testing were: MATLAB (version 7.12), Signal Processing Toolbox (version 6.15), Wavelet Toolbox (version 4.7), Image Processing Toolbox (version 7.2), Time-Frequency Toolbox (version 1.0) (http://tttb.nongnu.org/).

All testing was accomplished on a desktop computer (HP Compaq, 2.5GHz processor, AMD Athlon 64X2 Dual Core Processor 4800+, 2.00GB Memory (RAM), 32 Bit Operating System).

Testing was performed for 2 different triangular modulated FMCW waveforms. For each waveform, parameters were chosen for academic validation of signal processing techniques. Due to computer processing resources they were not meant to represent real-world values. The number of samples for each test was chosen to be either 256 or 512, which seemed to be the optimum size for the desktop computer. Testing was performed at three different SNR levels: $10 \mathrm{~dB}, 0 \mathrm{~dB}$, and the lowest SNR at which the signal could be detected. The noise added was white Gaussian noise, which best reflects the thermal noise present in the IF section of an intercept receiver [PAC09]. Kaiser windowing was used, when windowing was applicable. 50 runs were performed for each test, for statistical purposes. The plots included in this paper were done at a threshold of $5 \%$ of the maximum intensity and were linear scale (not dB) of analytic (complex) signals; the color bar represented intensity. The signal processing tools used for each task were the Spectrogram and the Scalogram.

Task 1 consisted of analyzing a triangular modulated FMCW signal (most prevalent LPI radar waveform [LIA09]) whose parameters were: sampling frequency $=4 \mathrm{KHz}$; carrier frequency $=1 \mathrm{KHz}$; modulation bandwidth $=500 \mathrm{~Hz}$; modulation period $=.02 \mathrm{sec}$.

Task 2 was similar to Task 1, but with different parameters: sampling frequency $=6 \mathrm{KHz}$; carrier frequency $=1.5 \mathrm{KHz}$; modulation bandwidth $=2400 \mathrm{~Hz}$; modulation period $=.15 \mathrm{sec}$. The different parameters were chosen to see how the different shapes/heights of the triangles of the triangular modulated FMCW would affect the metrics.

After each particular run of each test, metrics were extracted from the time-frequency representation.

The different metrics extracted were as follows:

1) Plot (processing) time: Time required for plot to be displayed. 
2) Percent detection: Percent of time signal was detected - signal was declared a detection if any portion of each of the signal components (4 chirp components for triangular modulated FMCW) exceeded a set threshold (a certain percentage of the maximum intensity of the time-frequency representation).
Threshold percentages were determined based on visual detections of low SNR signals (lowest SNR at which the signal could be visually detected in the timefrequency representation) (see Figure 1).

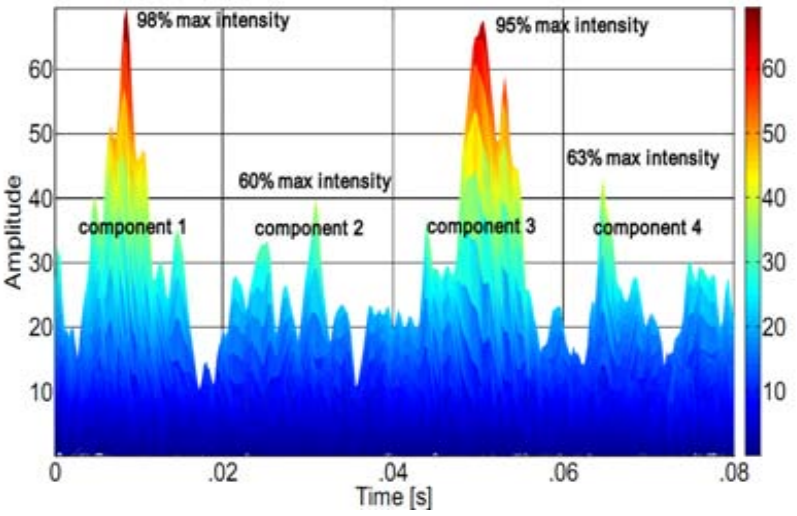

Figure 1: Threshold percentage determination. This plot is an amplitude vs. time ( $x-z$ view) of the Spectrogram of a triangular modulated FMCW signal (256 samples, with $S N R=-3 d B$ ). For visually detected low SNR plots (like this one), the percent of max intensity for the peak z-value of each of the signal components (the 2 legs for each of the 2 triangles of the triangular modulated FMCW) was noted (here $98 \%, 60 \%, 95 \%, 63 \%$ ), and the lowest of these 4 values was recorded (60\%). Ten test runs were performed for both time-frequency analysis tools (Spectrogram and Scalogram) for this waveform. The average of these recorded low values was determined and then assigned as the threshold for that particular time-frequency analysis tool. Note - the threshold for the Spectrogram is 60\%.

Thresholds were assigned as follows: automatically during the plotting process. From the Spectrogram (60\%); Scalogram (50\%). threshold plot, the signal was declared a detection if any

For percent detection determination, these threshold values were included in the time-frequency plot algorithms so that the thresholds could be applied portion of each of the signal components was visible (see Figure 2).

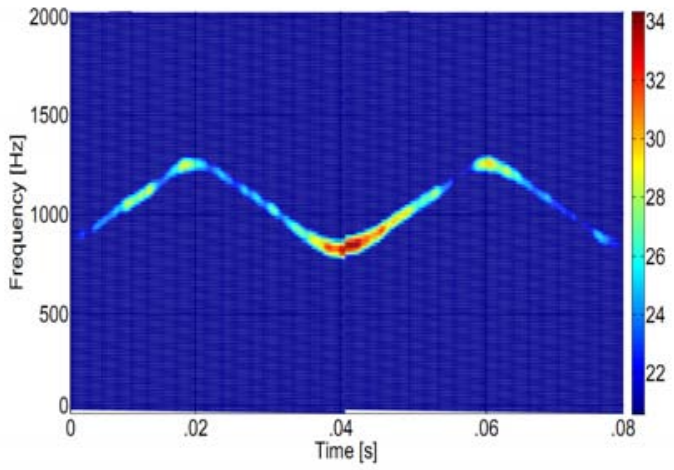

Figure 2: Percent detection (time-frequency). This plot is an frequency vs. time ( $x-y$ view) of the Spectrogram of a triangular modulated FMCW signal (256 samples, with SNR=10dB) with threshold value automatically set to 60\%. From this threshold plot, the signal was declared a (visual) detection because at least a portion of each of the 4 signal components (the 2 legs for each of the 2 triangles of the triangular modulated FMCW) was visible.

3) Carrier frequency: The frequency corresponding to the maximum intensity of the time-frequency representation (see Figure 3). 


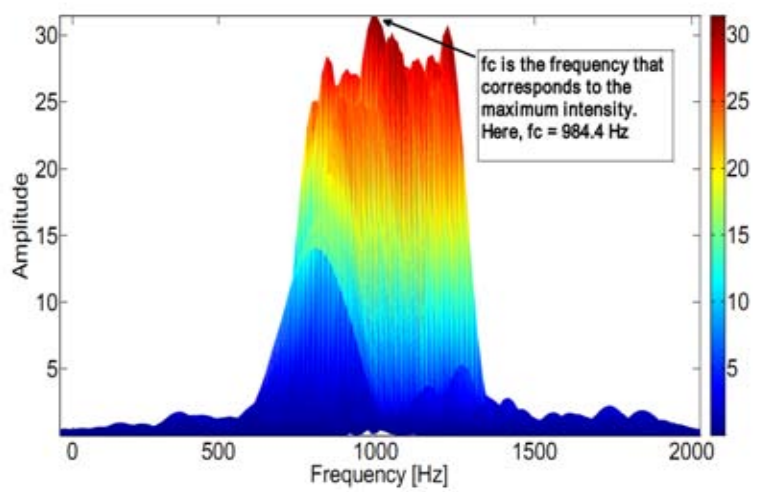

Figure 3: Determination of carrier frequency. Spectrogram of a triangular modulated FMCW signal (256 samples, $S N R=10 \mathrm{~dB})$. From the frequency-intensity $(y-z)$ view, the maximum intensity value is manually determined. The frequency corresponding to the max intensity value is the carrier frequency (here $\mathrm{fc}=984.4 \mathrm{~Hz}$ ).

4) Modulation bandwidth: Distance from highest frequency value of signal (at a threshold of $20 \%$ maximum intensity) to lowest frequency value of signal (at same threshold) in Y-direction (frequency).

The threshold percentage was determined based on manual measurement of the modulation bandwidth of the signal in the time-frequency representation. This was accomplished for ten test runs of each time-frequency analysis tool (Spectrogram and Scalogram), for each of the 2 waveforms. During each manual measurement, the max intensity of the high and low measuring points was recorded. The average of the max intensity values for these test runs was $20 \%$. This was adopted as the threshold value, and is representative of what is obtained when performing manual measurements. This $20 \%$ threshold was also adapted for determining the modulation period and the time-frequency localization (both are described below).

For modulation bandwidth determination, the $20 \%$ threshold value was included in the time-frequency plot algorithms so that the threshold could be applied automatically during the plotting process. From the threshold plot, the modulation bandwidth was manually measured (see Figure 4).

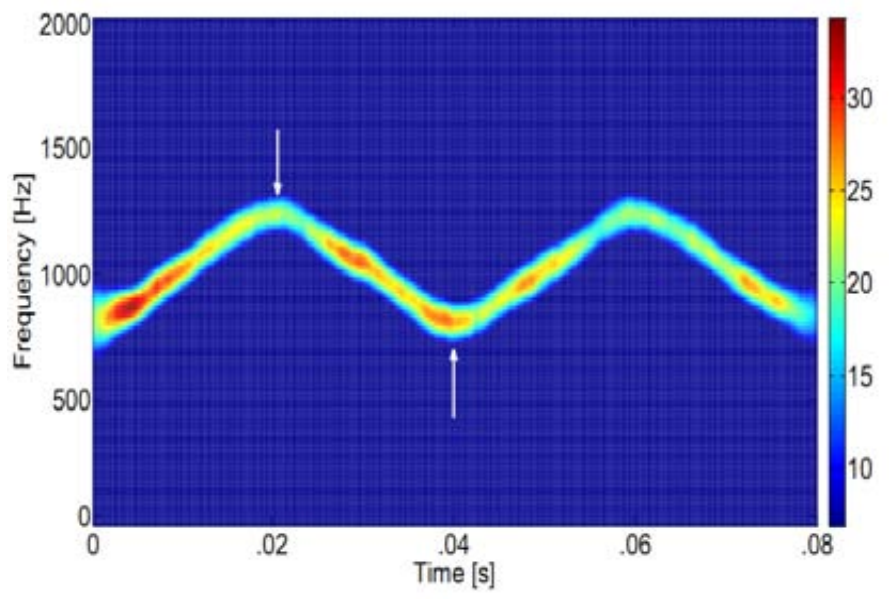

Figure 4: Modulation bandwidth determination. Spectrogram of a triangular modulated FMCW signal (256 samples, $S N R=10 \mathrm{~dB}$ ) with threshold value automatically set to $20 \%$. From this threshold plot, the modulation bandwidth was measured manually from the highest frequency value of the signal (top white arrow) to the lowest frequency value of the signal (bottom white arrow) in the y-direction (frequency)

5) Modulation period: Distance from highest frequency value of signal (at a threshold of $20 \%$ maximum intensity) to lowest frequency value of signal (at same threshold) in X-direction (time).

For modulation period determination, the $20 \%$ threshold value was included in the time-frequency plot algorithms so that the threshold could be applied automatically during the plotting process. From the threshold plot, the modulation period was manually measured (see Figure 5). 


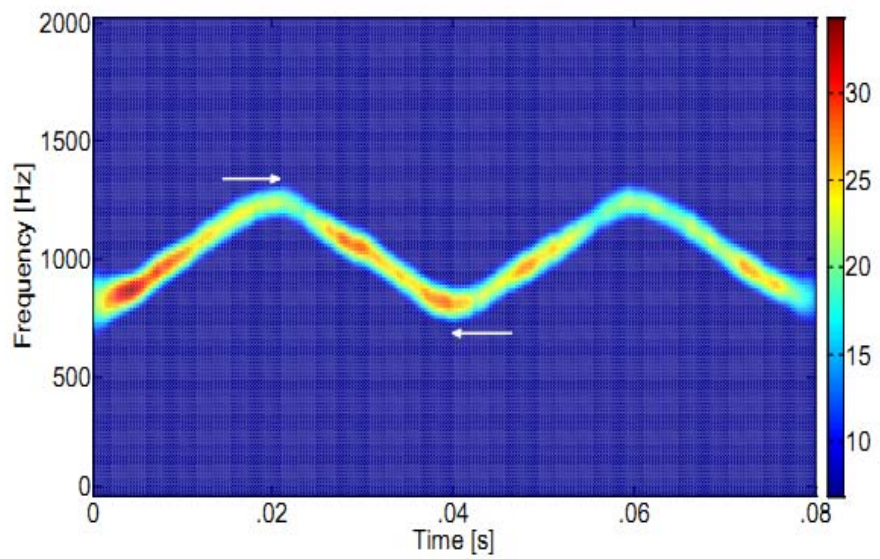

Figure 5: Modulation period determination. Spectrogram of a triangular modulated FMCW signal (256 samples, $S N R=10 \mathrm{~dB}$ ) with threshold value automatically set to $20 \%$. From this threshold plot, the modulation period was measured manually from the highest frequency value of the signal (top white arrow) to the lowest frequency value of the signal (bottom white arrow) in the $\mathrm{x}$-direction (time)

6) Time-frequency localization: Measure of the thickness of a signal component (at a threshold of $20 \%$ maximum intensity on each side of the component) - converted to \% of entire X-Axis, and $\%$ of entire Y-Axis.

frequency plot algorithms so that the threshold could be applied automatically during the plotting process. From the threshold plot, the time-frequency localization was manually measured (see Figure 6).

For time-frequency localization determination, the $20 \%$ threshold value was included in the time-

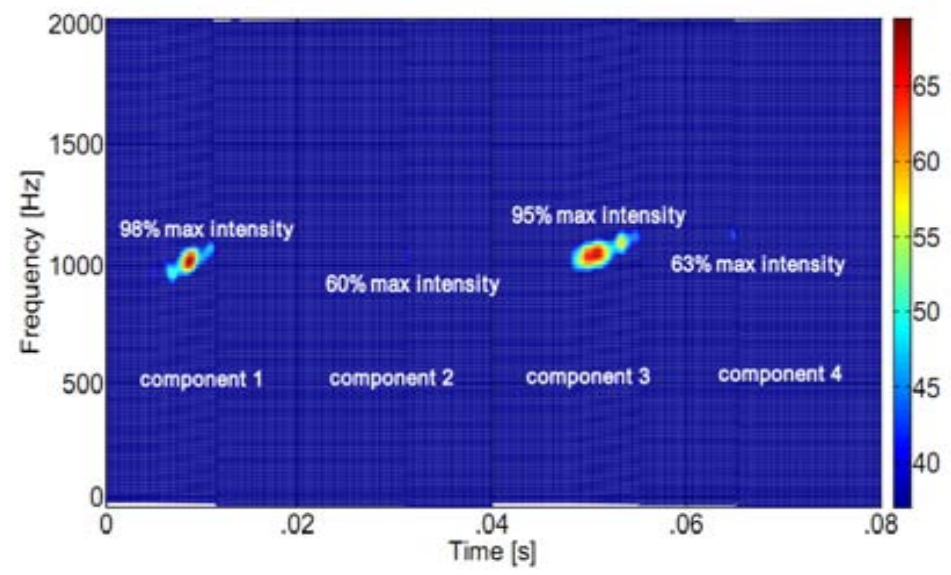

Figure 6: Time-frequency localization determination. Spectrogram of a triangular modulated FMCW signal (256 samples, $\mathrm{SNR}=10 \mathrm{~dB}$ ) with threshold value automatically set to $20 \%$. From this threshold plot, the time-frequency localization was measured manually from the left side of the signal (left white arrow) to the right side of the signal (right white arrow) in both the $\mathrm{x}$-direction (time) and the $\mathrm{y}$-direction (frequency). Measurements were made at the center of each of the 4 'legs', and the average values were determined. Average time and frequency 'thickness' values were then converted to: $\%$ of entire $x$-axis and $\%$ of entire $y$-axis.

7) Chirp rate: (modulation bandwidth)/(modulation period)

8) Lowest detectable SNR: The lowest SNR level at which at least a portion of each of the signal components exceeded the set threshold listed in the percent detection section above.

For lowest detectable SNR determination, these threshold values were included in the time-frequency plot algorithms so that the thresholds could be applied automatically during the plotting process. From the threshold plot, the signal was declared a detection if any portion of each of the signal components was visible. The lowest SNR level for which the signal was declared a detection is the lowest detectable SNR (see Figure 7). 


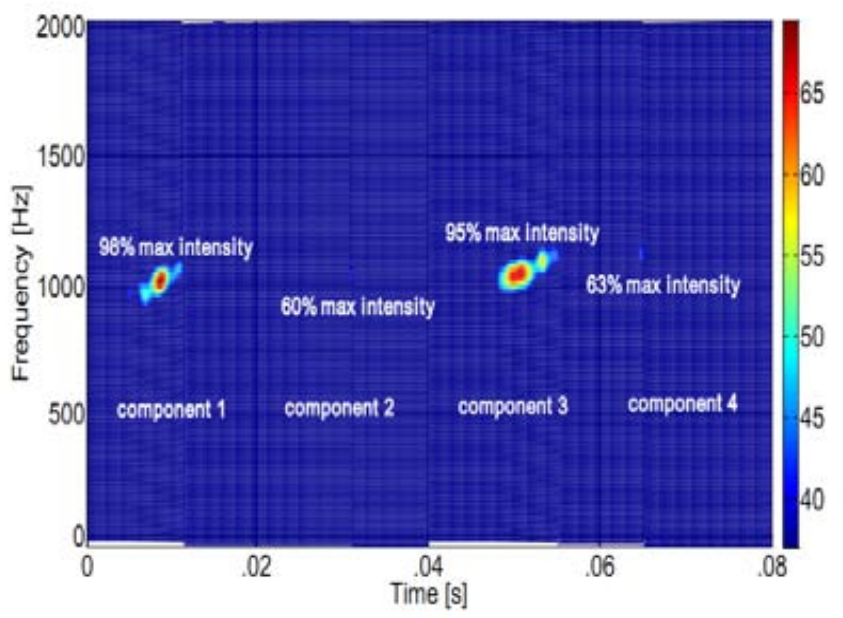

Figure 7: Lowest detectable SNR. This plot is an frequency vs. time ( $\mathrm{x}-\mathrm{y}$ view) of the Spectrogram of a triangular modulated FMCW signal (256 samples, with $S N R=-3 \mathrm{~dB}$ ) with threshold value automatically set to $60 \%$. From this threshold plot, the signal was declared a (visual) detection because at least a portion of each of the 4 signal components (the 2 legs for each of the 2 triangles of the triangular modulated FMCW) was visible. Note that the signal portion for the $60 \%$ max intensity (just above the ' $x$ ' in ' $m a x$ ') is barely visible, because the threshold for the Spectrogram is $60 \%$. For this case, any lower SNR would have been a non-detect. Compare to Figure 2, which is the same plot, except that it has an SNR level equal to 10dB.

The data from all 50 runs for each test was used to produce the actual, error, and percent error for each of these metrics listed above.

The metrics from the Spectrogram were then compared to the metrics from the Scalogram. By and large, the Spectrogram outperformed the Scalogram, as will be shown in the results section.

Table 1: Overall test metrics (average percent error: carrier frequency, modulation bandwidth, modulation period, chirp rate; average: percent detection, lowest detectable snr, plot time, time-frequency localization (as a percent of $x$ axis and y axis) for the two classical time-frequency analysis techniques (Spectrogram versus Scalogram).

\begin{tabular}{|l|c|c|}
\hline parameters & Spectrogram & Scalogram \\
\hline carrier frequency & $6.83 \%$ & $28.26 \%$ \\
\hline modulation bandwidth & $16.60 \%$ & $0.72 \%$ \\
\hline modulation period & $0.68 \%$ & $28.47 \%$ \\
\hline chirp rate & $16.25 \%$ & $62.22 \%$ \\
\hline percent detection & $70.0 \%$ & $-2.67 \mathrm{db}$ \\
\hline lowest detectable snr & $-3.67 \mathrm{db}$ & $4.16 \mathrm{~s}$ \\
\hline plot time & $3.28 \mathrm{~s}$ & $4.51 \%$ \\
\hline time-frequency localization-x & $2.88 \%$ & $9.0 \%$ \\
\hline time-frequency localization-y & $5.75 \%$ & $2 \%$ \\
\hline
\end{tabular}

From Table 1, the Spectrogram outperformed the Scalogram in every metrics category.

\section{RESULTS}

Table 1 presents the overall test metrics for the two classical time-frequency analysis techniques used in this testing (Spectrogram versus Scalogram).
Figure 8 shows comparative plots of the Spectrogram (left) vs. the Scalogram (right) (triangular 
modulated FMCW signal - Task 1) at SNRs of $10 \mathrm{~dB}$ (top), OdB (middle), and -3dB (bottom).
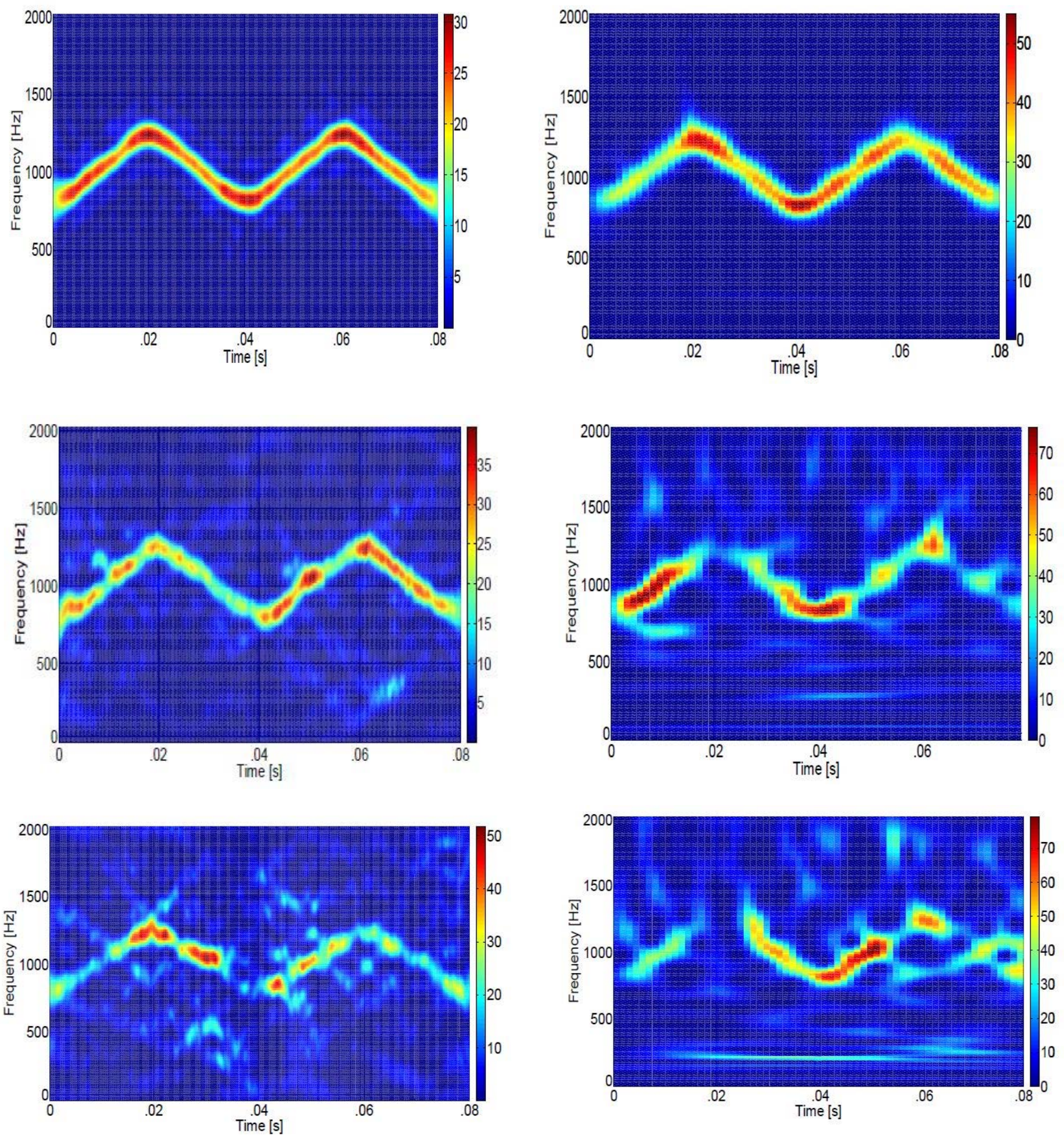

Figure 8: Comparative plots of the triangular modulated FMCW (task 1) low probability of intercept radar signals (Spectrogram (left-hand side) vs. the Scalogram (right-hand side)). The SNR for the top row is 10dB, for the middle row is $0 \mathrm{~dB}$, and for the bottom row is $-3 \mathrm{~dB}$. In general, the Spectrogram signals appear more localized ('thinner') than do the Scalogram signals. In addition, the Spectrogram signals appear more readable than the Scalogram signals at every SNR level.

Figure 9 shows comparative plots of the Spectrogram (left) vs. the Scalogram (right) (triangular modulated FMCW signal - Task 2) at SNRs of $10 \mathrm{~dB}$ (top), OdB (middle), and -3dB (bottom). 

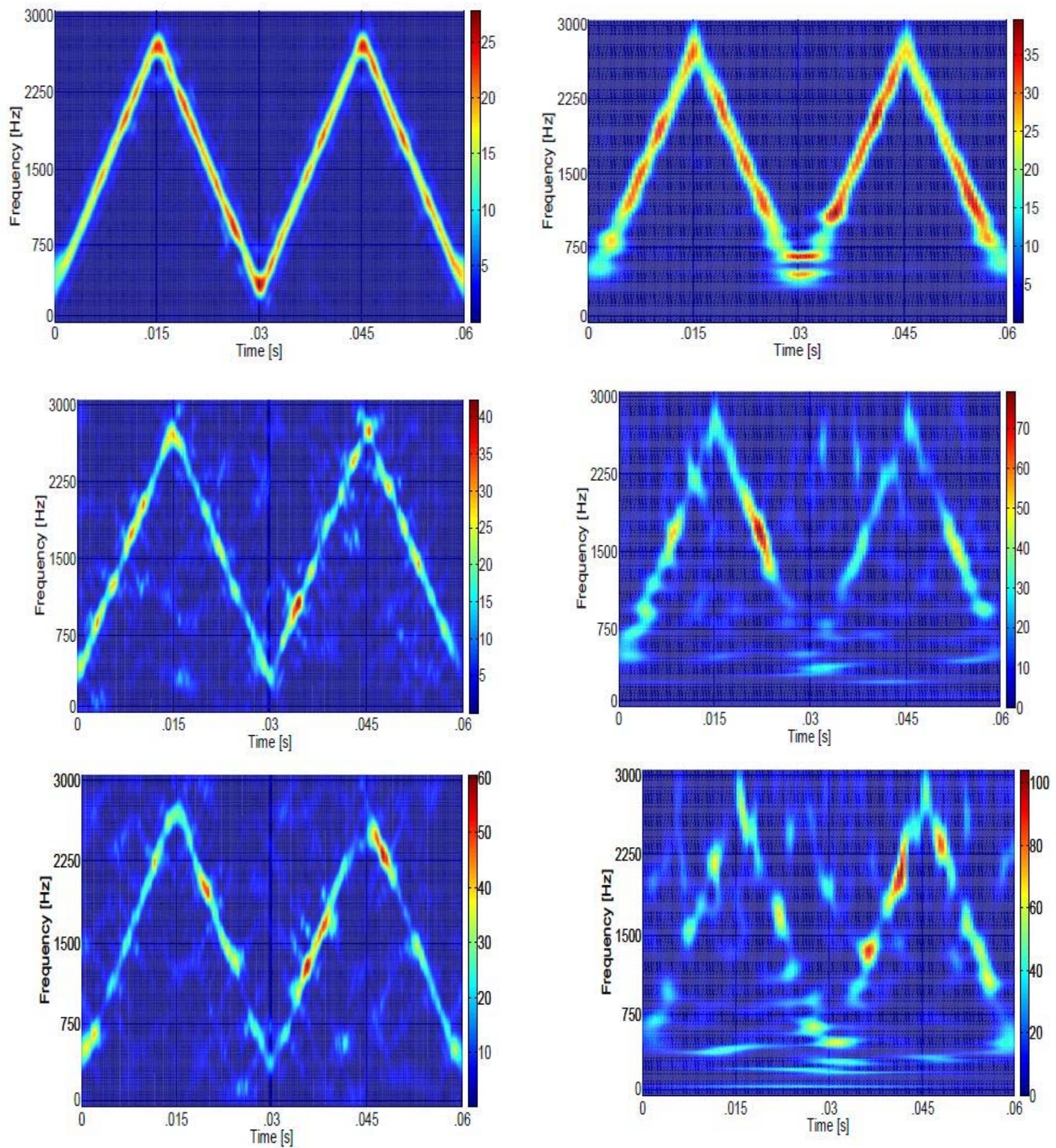

Figure 9: Comparative plots of the triangular modulated FMCW (task 2) low probability of intercept radar signals (Spectrogram (left-hand side) vs. the Scalogram (right-hand side)). The SNR for the top row is 10dB, for the middle row is $0 \mathrm{~dB}$, and for the bottom row is $-3 \mathrm{~dB}$. In general, the Spectrogram signals appear more localized ('thinner') than do the Scalogram signals. In addition, the Spectrogram signals appear more readable than the Scalogram signals at every SNR level.

\section{Discussion}

This section will elaborate on the results from the previous section.

From Table 1, the Spectrogram outperformed the Scalogram in every category. The Spectrogram's reduction of cross-term interference is grounds for its better plot time. Average percent detection and lowest detectable SNR are both based on visual detection in the Time-Frequency representation. Figure 8 and Figure 9 show clearly that the signals in the Spectrogram plots are more readable than those in the Scalogram plots, which account for the Spectrogram's better average percent detection and lowest detectable SNR. At relatively low frequencies (as in this paper), wavelets (Scalograms), because of their multi-resolution analysis basis, are better resolved (localized) in frequency and 
more poorly resolved (localized) in time. Therefore for relatively low frequencies, the best waveforms to be analyzed by wavelets (Scalograms) are tonals. In addition, the irregularity of the wavelet (Scalogram) basis lends itself to analysis of signals with discontinuities (such as frequency hopping signals (tonals)). Also, since the wavelet is irregular in shape and compactly supported, it makes it an ideal tool for analyzing signals of transient nature (such as the frequency hopping signals (tonals)). Therefore as the signal goes from being 'flat' (i.e. a tonal) signal, to more 'upright' (i.e. a triangular modulated FMCW) signal, the Scalogram of this signal becomes more poorly resolved (localized), i.e. 'fatter', accounting for the Scalogram's poorer metrics in the categories of modulation bandwidth, modulation period, chirp rate, carrier frequency, time-frequency localization $(x)$, and timefrequency localization (y)

\section{Conclusions}

Digital intercept receivers, whose main job is to detect and extract parameters from low probability of intercept radar signals, are currently moving away from Fourier-based analysis and towards classical timefrequency analysis techniques, such as the Spectrogram, and Scalogram, for the purpose of analyzing low probability of intercept radar signals. Based on the research performed for this paper (the novel direct comparison of the Spectrogram versus the Scalogram for the signal analysis of low probability of intercept triangular modulated FMCW radar signals) it was shown that the Spectrogram by-and-large outperformed the Scalogram for analyzing these low probability of intercept radar signals - for reasons brought out in the discussion section above. More accurate characterization metrics could well translate into saved equipment and lives.

Future plans include continuing to analyze low probability of intercept radar waveforms (such as the frequency hopping and the triangular modulated FMCW), using additional time-frequency analysis techniques.

\section{References Références Referencias}

1. [ALN00] Al-Namiy, F., Nigam, M., On the Design of 2-Band FIR QMF Filter Banks Using WLS Techniques. Proceedings of the Fourth IEEE International Conference on High Performance Computing in the Asia-Pacific Region, Vol. 2, pp. 772-776, May 2000.

2. [ANJ09] Anjaneyulu, L., Murthy, N., Sarma, N., A Novel Method for Recognition of Modulation Code of LPI Radar Signals. International Journal of Recent Trends in Engineering, Vol. 1, No. 3, pp. 176-180, May 2009.
3. [BOA03] Boashash, B., Time Frequency Signal Analysis and Processing: A Comprehensive Reference. Elsevier, Oxford, England, 2003.

4. [CHE09] Chen, C., He, M., Jin, H., Li, H., Shen, Z., A Novel Method for Extraction of In-Pulse Feature of Multi-Component LFM Signal. ICEMI 2009, pp. 4-627 - 4-630, 2009.

5. [COH02] Cohen, L., The Wavelet Transform and Time-Frequency Analysis. in: Debnath, L., Wavelet Transforms and Signal Processing, Birkhauser, pp. 3-22, 2002.

6. [COH95] Cohen, L., Time-Frequency Analysis. Prentice Hall, Upper Saddle River, NJ, 1995.

7. [FAR96] Farrell, T.C., Prescott, G., A Low Probability of Intercept Signal Detection Receiver Using Quadrature Mirror Filter Bank Trees. IEEE Proceedings, pp. 1558-1561, 1996.

8. [FAR99] Farrell, T., Prescott, G., A Method for Finding Orthogonal Wavelet Filters with Good Energy Tiling Characteristics. IEEE Transactions on Signal Processing, Vol. 47, No. 1, pp. 220-223, Jan. 1999.

9. [GAL05] Galleani, L., Cohen, L., Noga, A., A Time-Frequency Approach to the Adjustable Bandwidth Concept. Digital Signal Processing, Vol. 16, Issue 5, pp. 454-467, Sept. 2006.

10. [GOH00] Goh, C., Lim, T., A WLS Algorithm for the Design of Low-Delay Quadrature Mirror Filter Banks. Proceedings of the IEEE International Symposium on Circuits and Systems, Vol. 1, pp. 615-618, May 2000.

11. [GRI07] Grishin, Y., Janczak, D., ComputerAided Methods of the LPI Radar Signal Detection and Classification. Proc. of SPIE Vol. 6937, pp. 1-5, 2007.

12. [GRI08] Grishin, Y., Interferences Excision Via Time-Frequency Distribution in Radio Communication Systems. EMD 2008, XVIII-th International Conference on Electromagnetic Disturbances, Vilnius, Lithuania, pp. 85-88, 25-26 September 2008.

13. [GUL08] Gulum, T., Pace, P., Cristi, R., Extraction of Polyphase Radar Modulation Parameters Using a Wigner-Ville Distribution-Radon Transform. IEEE International Conference on Acoustics, Speech, and Signal Processing, Las Vegas, NV, April 2008.

14. [HAN00] Han, S., Hong, H., Seo, D., Choi, J., Target Position Extraction Based on Instantaneous Frequency Estimation in a Fixed-Reticle Seeker. Opt. Eng., Vol. 39, pp. 2568-2573, September 2000.

15. [HIP00] Hippenstiel, R., Fargues, M., Moraitakis, I., Williams, C., Detection and Parameter Estimation of Chirped Radar Signals. Final Report, Naval Postgraduate School, Monterey, CA, Jan. 10, 2000. 
16. [HLA92] Hlawatsch, F., Boudreaux-Bartels, G.F., Linear and Quadratic Time-Frequency Signal Representations. IEEE Signal Processing Mag., Vol. 9, No. 2, pp. 21-67, April 1992.

17. [ISI96] Auger, F., Flandrin, P., Goncalves, P., Lemoine, O., Time-Frequency Toolbox Users Manual. Centre National de la Recherche Scientifique and Rice University, 1996.

18. [LAR92] Lari, F., Zakhor, A., Automatic Classification of Active Sonar Data Using TimeFrequency Transforms. Proceedings of IEEE-SP International Symposium on Time-Frequency and Time-Scale Analysis, Victoria, BC, pp. 21-24, Oct. 46, 1992.

19. [LIA09] Liang, Y., Zhang, L., Xing, M., Bao, Z., High-Speed Ground Moving Target Detection Research Using Triangular Modulation FMCW. Front. Electr. Electron. Eng., China, 4(2), pp. 127133, 2009.

20. [LIW08] Li, W., Dan, M., Wang, X., Li, D., Wang, G., Fast Estimation Method and Performance Analysis of Frequency Modulation Rate Via RAT. Proceedings of the 2008 IEEE International Conference on Information and Automation, Zhangjiajie, China, pp. 144-147, June 20-23, 2008.

21. [LIX08] Li, X., Bi, G., A New Reassigned TimeFrequency Representation. 16th European Signal Processing Conference, Lausanne, Switzerland, pp. 1-4, August 25-29, 2008.

22. [LIY03] Li, Y., Xiao, $X .$, Recursive Filtering Radon-Ambiguity Transform Algorithm for Detecting Multi-LFM Signals. Journal of Electronics (China), Vol. 20, No. 3, pp. 161-166, May 2003.

23. [MIL02] Milne, P., Pace, P., Wigner Distribution Detection and Analysis of FMCW and P-4 Polyphase LPI Waveforms. Proceedings of ICASSP, Orlando, FL, pp. 3944-3947, 2002.

24. [MIT01] Mitra, S., Digital Signal Processing, A Computer-Based Approach, Second Edition. McGraw-Hill, Boston, MA, 2001.

25. [OZD03] Ozdemir, A., Time-Frequency Component Analyzer. Dissertation, Bilkent University, Ankara, Turkey, Sept. 2003.

26. [PAC09] Pace, P., Detecting and Classifying Low Probability of Intercept Radar. Artech House, Norwood, MA, 2009.

27. [PAP95] Papandreou, A., Boudreaux-Bartels, G.F., Kay, S., Detection and Estimation of Generalized Chirps Using Time-Frequency Representations. 1994 Conference Record of the Twenty-Eighth Asilomar Conference on Signals, Systems and Computers, pp. 50-54, 1994.

28. [RAN01] Rangayyan, R., Krishnan, S., Feature Identification in the Time-Frequency Plane by Using the Hough-Radon Transform. Pattern Recognition, Vol. 34, pp. 1147-1158, 2001.
29. [SAR98] Sarkar, T., Su, C., et al., A Tutorial on Wavelets from an Electrical Engineering Perspective, Part 1: Discrete Wavelet Techniques. IEEE Antennas and Propagation Magazine, Vol. 40, No. 5, pp. 49-70, October 1998.

30. [SAT98] Sarkar, T., Su, C., A Tutorial on Wavelets from an Electrical Engineering Perspective, Part 2: The Continuous Case. IEEE Antennas and Propagation Magazine, Vol. 40, No. 6, pp. 36-49, December 1998.

31. [WAJ08] Wang, Y., Jiang, Y., Detection and Parameter Estimation of Multicomponent LFM Signal Based on the Cubic Phase Function. EURASIP Journal on Advances in Signal Processing, Vol. 2008, Article ID 743985, pp. 1-7, 2008

32. [WAN10] Wang, P., Li, H., Djurovic, I., Himed, B., Integrated Cubic Phase Function for Linear FM Signal Analysis. IEEE Transactions on Aerospace and Electronic Systems, to appear, 2010.

33. [WEI03] Wei, G., Wu, S., Mao, E., Analysis of Multicomponent LFM Signals Using TimeFrequency and The Gray-Scale Inverse Hough Transform. IEEE Workshop on Statistical Signal Processing, pp. 190-193, September 28 - October 1, 2003.

34. [WIL06] Wiley, R., ELINT: The Interception and Analysis of Radar Signals. Artech House, Norwood, MA, 2006.

35. [WON09] Wong, K., Davidson, T., Abelkader, S., Detection of Low Probability of Intercept Radar Signals. Defence R\&D Canada - Ottawa Contract Report 2009-142, September 2009.

36. [XIA99] Xia, X., Chen, V., A Quantitative SNR Analysis for the Pseudo Wigner-Ville Distribution. IEEE Transactions on Signal Processing, Vol. 47, No. 10, pp. 2891-2894, October, 1999.

37. [ZAH99] Zahhad, A., Sabah, M., Design of Selective M-Channel Perfect Reconstruction FIR Filter Banks. IEE Electronics Letters, Vol. 35, No. 15, pp. 1223-1225, 1999.

38. [ZHA00] Zhang, Z., Jiao, L., A Simple Method for Designing Pseudo QMF Banks. Proceedings of the IEEE International Conference on Communication Technology, Vol. 2, pp. 1538-1541, Aug. 2000. 
